\title{
Chronic Miliarial Gout Associated with Carpal Tunnel Syndrome: A Very Rare Clinical Presentation
}

\author{
Victoria Sadovici-Bobeica $^{1}$, Lucia Mazur-Nicorici' ${ }^{1}$, Aliona Nicorici² ${ }^{2}$ Virginia Șalaru ${ }^{3}$, Natalia Loghin-Oprea ${ }^{1}$, Maria Garabajiu $^{1}$, \\ Valeriu Istrati ${ }^{1}$, Minodora Mazur ${ }^{1}$ \\ ${ }^{1}$ Department of Internal Medicine, State University of Medicine and Pharmacy "Nicolae Testemițanu", Chisinau, Republic of Moldova \\ ${ }^{2}$ Laboratory of Immunology, Institute of Oncology, Chisinau, Republic of Moldova \\ ${ }^{3}$ Department of Family Medicine, State University of Medicine and Pharmacy "Nicolae Testemițanu", Chisinau, Republic of Moldova
}

\section{Doi: 10.12890/2018_000926 - European Journal of Case Reports in Internal Medicine - @ EFIM 2018}

\begin{abstract}
Received: $11 / 07 / 2018$
Accepted: $14 / 07 / 2018$

Published: 08/08/2018
\end{abstract} How to cite this article: Sadovici-Bobeica V, Mazur-Nicorici L, Nicorici A, Șalaru V, Loghin-Oprea N, Garabajiu M, Istrati V, Mazur M. Chronic miliarial gout
associated with carpal tunnel syndrome: a very rare clinical presentation. EJCRIM 2018;5 doi:10.12890/2018_000926.

Conflicts of Interests: The authors declare that they have no competing interests.

This article is licensed under a Commons Attribution Non-Commercial 4.0 License

\section{ABSTRACT}

This case report describes an unusual form of gout, called miliarial gout, in association with carpal tunnel syndrome in a 54-year-old woman. Miliarial gout was first described in 2007 and is a very rare presentation of chronic tophaceous gout. The latter condition can cause carpal tunnel syndrome, but this association has not previously been described in association with miliarial gout. In addition, the authors discuss the use of the parsimony principle in internal medicine whereby a single cause is first sought for different symptoms presenting at the same time.

\section{LEARNING POINTS}

- Miliarial gout, a form of chronic tophaceous gout, is a very rare clinical presentation of the disease.

- Patients with miliarial gout, despite subcutaneous nodules, may present with other organ involvement.

- Carpal tunnel syndrome in patients with gout may be caused by monosodium urate crystal deposition.

\section{KEYWORDS}

Gout, miliary gout, carpal tunnel syndrome

\section{INTRODUCTION}

Monosodium urate crystals (MSU) are deposited in gout ${ }^{[1]}$, a disease which has a prevalence of $0.9-2.5 \%$ depending on the country ${ }^{[2]}$. Chronic tophaceous gout is a particular form of the disease that manifests as MSU deposition intradermally or within the subcutaneous tissues of the $\operatorname{skin}^{[3]}$. A new form of this condition, called miliarial gout, was described in 2007 and consists of multiple tiny intradermal papules containing a white to cream-coloured material and scattered on an erythematous base ${ }^{[4]}$. Several case reports have since described this particular form of gout, but to our knowledge, no association between miliarial gout and carpal tunnel syndrome has been previously reported.

\section{CASE DESCRIPTION}

A 54-year-old woman was referred for a rheumatology consultation by her family physician because of paraesthesia of the fingers of the right hand, which worsened at night and after physical activity. The symptoms had started 3 years previously and had a remitting/relapsing course, 
with significant aggravation during the preceding 3 months. A neurologist examined the patient and an EMG and echography confirmed the diagnosis of carpal tunnel syndrome. However, the condition worsened despite treatment with oral non-steroidal anti-inflammatory drugs. The anamnesis included menopause at the age of 49 years, no tobacco or alcohol consumption, no similar family history, and hypertension treated with beta-blockers, diuretics and aspirin.

The physical examination showed a patient in no distress, with normal vital signs (BP 123/80 mm/Hg, pulse 68/minute), a BMI of $29.6 \mathrm{~kg} /$ $\mathrm{m}^{2}$, no tender or swollen joints, and hyposensitivity of the first, second and third fingers of the right hand. Examination of the skin identified white non-tender maculopapular lesions on an erythematous base, with a diameter of $2-5 \mathrm{~mm}^{2}$ (Fig. 1).

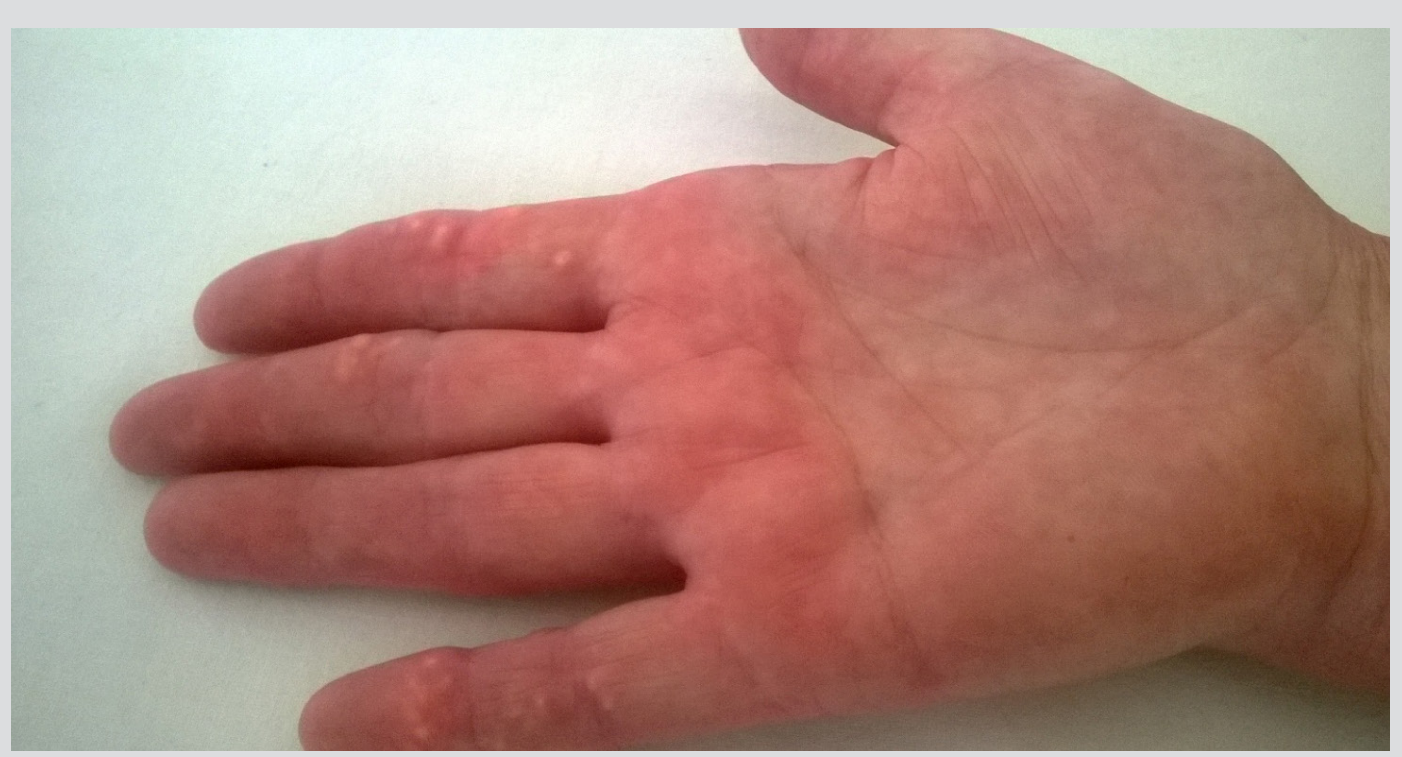

Figure 1. Miliarial gout manifested as white maculopapular lesions on an erythematous base, with a diameter of 2-5 $\mathrm{mm}^{2}$

Laboratory investigations confirmed a mild inflammatory syndrome with an erythrocyte sedimentation rate of $17 \mathrm{~mm} / \mathrm{h}$ (normal range $2-15$ $\mathrm{mm} / \mathrm{h}$ ), an increased C-reactive protein level of $12 \mathrm{mg} / \mathrm{dl}$ (normal range $<6 \mathrm{mg} / \mathrm{dl}$ ) and a mild decrease in the glomerular filtration rate (52 $\mathrm{ml} / \mathrm{min} / 1.73 \mathrm{~m}^{2}$ ). The patient's serum uric acid level was $6.1 \mathrm{mg} / \mathrm{dl}$. Morphological examination of material from the skin papules and carpal tunnel aspirate revealed MSU crystals, confirming the diagnosis (Figs. 2 and 3).

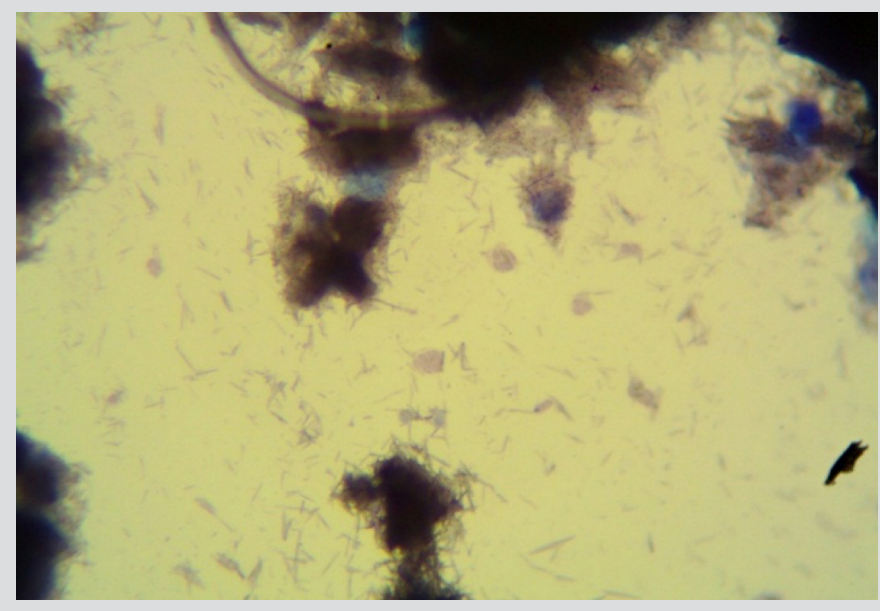

Figure 2. Morphological analysis of the content of a maculopapular skin lesion revealed groups of transparent anuclear structures, identified as monosodium urate acid crystals

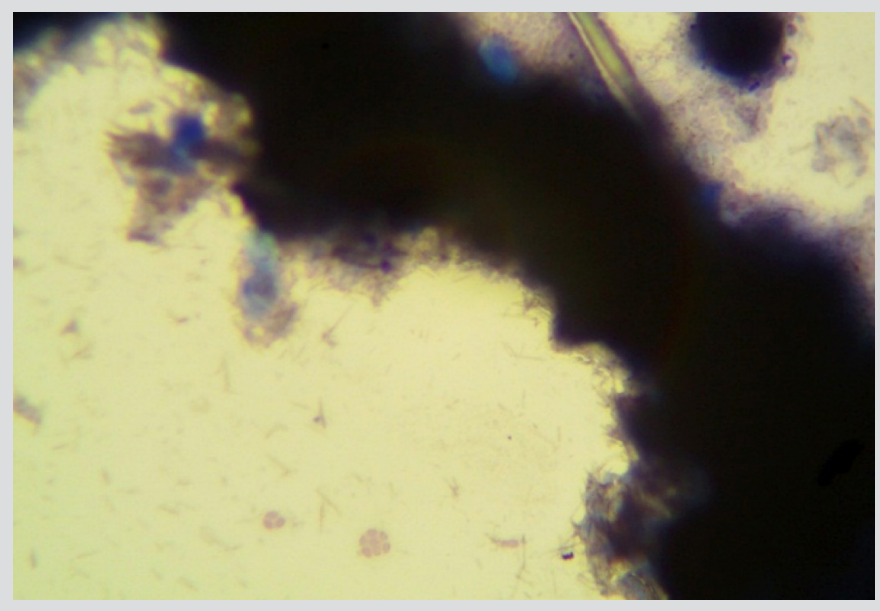

Figure 3. Morphological analysis of the right carpal tunnel aspirate revealed groups of transparent anuclear structures, identified as monosodium urate acid crystals 


\section{DISCUSSION}

As chronic tophaceous gout can cause carpal tunnel syndrome $\mathrm{e}^{[5]}$, miliarial gout, being a form of chronic tophaceous gout, could possibly result in the same condition, although no such cases have been previously described.

We diagnosed miliarial gout, an extremely rare form of chronic tophaceous gout, that had caused carpal tunnel syndrome in a patient with multiple risk factors for gout including overweight, a mildly decreased glomerular filtration rate, and aspirin and diuretic intake.

The patient was managed according to the EULAR evidence-based recommendations for gout ${ }^{[2]}$, which included lifestyle changes with diet modification and febuxostat therapy for hypouricaemia. The patient has stopped taking diuretics. A local corticosteroid injection was also administered, which resulted in significant regression of clinical symptoms. After 3 months of follow-up, the patient's uric acid level had dropped to $4.7 \mathrm{mg} / \mathrm{dl}$ and the symptoms had resolved.

\section{CONCLUSION}

The parsimony principle, also called Occam's razor, can be applied in the clinical setting when two clinical entities are present together. This principle was first described in 1347 and states that "Entities are not to be multiplied without necessity" (Non sunt multiplicanda entia sine necessitate). Applying the principle in medicine means a single cause is first sought even for multiple symptoms/syndromes. Application of the parsimony principle in our case resulted in the diagnosis of miliarial gout with carpal tunnel syndrome.

\section{REFERENCES}

1. Pascual E, Bardin T, Richette P. Crystal arthropathies. Gout. In: EULAR textbook on rheumatic diseases. BMJ, London; 2012, p. 301.

2. Richette P, Doherty M, Pascual E, et al. 2016 updated EULAR evidence-based recommendations for the management of gout. Ann Rheum Dis 2017;76:29-42.

3. Mireku K, Burgy J, Davis L. Miliarial gout: a rare clinical presentation. J Am Acad Dermatol 2014;71:e17-e18.

4. Shukla R, Vender RB, Alhabeeb A, Salama S, Murphy F. Miliarial gout (a new entity). J Cutan Med Surg 2007;11:31-34.

5. PubMed. https://www.ncbi.nlm.nih.gov/pubmed/?term=carpal+tunnel+gout 